Original research article

\title{
Reshaping cortical activity with subthalamic stimulation in Parkinson's disease during finger tapping and gait mapped by near infrared spectroscopy
}

\author{
Ondrej Klempir ${ }^{1 *}$, Radim Krupicka ${ }^{1}$, Jan Mehnert ${ }^{3,4}$, Vaclav Cejka ${ }^{1,2}$, Kamila Polakova ${ }^{2}$, \\ Hana Brozova ${ }^{2}$, Zoltan Szabo ${ }^{1}$, Evzen Ruzicka ${ }^{2}$, Robert Jech ${ }^{2}$ \\ ${ }^{1}$ Czech Technical University in Prague, Faculty of Biomedical Engineering, Department of Biomedical Informatics, Kladno, Czech Republic \\ ${ }^{2}$ Charles University, First Faculty of Medicine and General University Hospital, Department of Neurology, Prague, Czech Republic \\ ${ }^{3}$ University Medical Center Eppendorf, Department of Systems Neuroscience, Hamburg, Germany \\ ${ }^{4}$ Max Planck Institute for Human Cognitive and Brain Sciences, Leipzig, Germany
}

\begin{abstract}
Exploration of motor cortex activity is essential to understanding the pathophysiology in Parkinson's Disease (PD), but only simple motor tasks can be investigated using a fMRI or PET. We aim to investigate the cortical activity of PD patients during a complex motor task (gait) to verify the impact of deep brain stimulation in the subthalamic nucleus (DBS-STN) by using Near-Infrared-Spectroscopy (NIRS). NIRS is a neuroimaging method of brain cortical activity using low-energy optical radiation to detect local changes in (de)oxyhemoglobin concentration. We used a multichannel portable NIRS during finger tapping (FT) and gait. To determine the signal activity, our methodology consisted of a pre-processing phase for the raw signal, followed by statistical analysis based on a general linear model. Processed recordings from 9 patients were statistically compared between the on and off states of DBS-STN. DBS-STN led to an increased activity in the contralateral motor cortex areas during FT. During gait, we observed a concentration of activity towards the cortex central area in the "stimulation-on" state. Our study shows how NIRS can be used to detect functional changes in the cortex of patients with PD with DBS-STN and indicates its future use for applications unsuited for PET and a fMRI.
\end{abstract}

Keywords: Deep brain stimulation; Gait; Near infrared spectroscopy; Neuroinformatics; Parkinson's disease; Subthalamic nucleus

Highlights:

- Our study shows how fNIRS can be used to detect functional changes in the motor cortex with DBS-STN;

- DBS-STN led to an increased activity in the contralateral motor cortex areas during finger tapping;

- This is the first study to investigate gait in Parkinson's disease with DBS using multichannel fNIRS imaging.

\section{Introduction}

Functional Near-Infrared-Spectroscopy (fNIRS) equipment has become commercially available and widely used in clinical practice such as diagnosis in psychiatry, neurosurgery or pediatrics (Kim et al., 2017). The continuous-wave near-infrared spectroscopy has made it possible to measure hemodynamic change in the living tissue, or more precisely the product of change in hemoglobin concentration in living tissue and the mean optical path length. This equipment helps physicians to obtain physiologically useful information in the human body with a noninvasive approach, e.g. monitoring oxygen metabolism in the muscles (Boushel and Piantadosi, 2000). NIRS is also a portable neuroimaging method that uses low-energy op- tical radiation to measure extinction properties in subsurface tissue caused by concentration changes of hemoglobin which correlates with cortical activity (Torricelli et al., 2014). Motor cortex activity, therefore, is a key to understanding the pathophysiological mechanisms of movement control in Parkinson's disease (PD), as well as the function of its treatment. However, functional brain imaging with magnetic resonance (fMRI) is not feasible during gait (Gilat et al., 2015). Gait disorders seriously affect the functional state and quality of life of patients with PD. With time, about $50-70 \%$ of patients develop gait and balance disorders and show freezing of gait (FOG) which is characterized by brief episodes of inability to step and lift their feet from the ground or by extremely short steps (Varriale et al., 2018). Deep brain stimulation of the subthalamic nucleus (DBS-STN) has proven to be effective for bradykinesia, tremor, dystonia and gait disorder in PD (Huang et al., 2018).

\footnotetext{
* Author for correspondence: Ondrej Klempir, Czech Technical University in Prague, Faculty of Biomedical Engineering, Department of Biomedical Informatics, nam. Sitna 3105, 27201 Kladno, Czech Republic; e-mail: klempond@gmail.com http://doi.org/10.32725/jab.2019.014

Submitted: 2018-05-30 • Accepted: 2019-08-19 • Prepublished online: 2019-09-11

J Appl Biomed 17/3: 157-166 • EISSN 1214-0287 • ISSN 1214-021X

(c) 2019 The Authors. Published by University of South Bohemia in České Budějovice, Faculty of Health and Social Sciences.

This is an open access article under the CC BY-NC-ND license.
} 
The NIRS method is based on measuring the extinction of hemoglobin for two wavelengths: oxyhemoglobin (HbO, oxy$\mathrm{Hb}$ ) and deoxyHb using optoelectrodes, which are placed into the grid as EEG electrodes (Giacometti and Diamond, 2013). The hemodynamic response (HR) is similar to the fMRI (Cui et al., 2011). The HR starts with a latency of 1-2 seconds after the beginning of the event and reaches a maximum after 7 seconds. A frequency band associated with the event corresponds approximately to the range of the HR recorded using a blood-oxygen-level-dependent (BOLD) fMRI in the range of $0.01 \mathrm{~Hz}$ to $0.1 \mathrm{~Hz}$ (Tohka et al., 2008). Motor activity in a fMRI is typically around the frequency of $0.04 \mathrm{~Hz}$, which agrees with the measured frequency of the NIRS signal during a simple motor task (dominance at $0.035 \mathrm{~Hz}$ ). In comparison to a fMRI, NIRS provides lower spatial resolution, but has a better time resolution. It has been shown that there is a significant correlation in a time series NIRS and fMRI (Strangman et al., 2002). The depth of penetration in the head of an adult is in the range of $15-25 \mathrm{~mm}$. The NIRS system is safe, versatile, portable and can measure from anywhere on the head (e.g. prefrontal cortex, motor cortex, parietal cortex).

The purpose of this study is to verify the applicability of fNIRS to detect changes in motor cortex activity during gait in PD patients treated with bilateral DBS-STN and to explore the neuronal network involved.

\section{Related work}

Previous fMRI/PET studies have shown many types of motor activations in healthy controls (HC subjects) and PD patients. Self-initiated (18 PD patients) finger tapping (FT) activated the left primary motor cortex (M1), the premotor cortex (PMC), the pre-supplementary motor area (SMA) and the parietal cortex (PC) (Wu et al., 2011). Relatively decreased activities in the rostral part of the SMA and in the right dorsolateral prefrontal cortex (PFC) during sequential motor task were demonstrated when comparing 6 patients with PD with 6 HC subjects (Sabatini et al., 2000). Neuronal activity is modulated by the amplitude and the frequency of the tapping (Blinkenberg et al., 1996; Kawashima et al., 1999; Rao et al., 1996; Sadato et al., 1996). PD patients, after Levodopa drug administration, showed significant differences in the motor cortico-striatal network (motor cortex, PMC, SMA, putamen and thalamus) and the cerebellum while using their more affected hand during FT (Martinu et al., 2014). In a PET study, motor tasks in PD patients induced hypoactivation in the SMA during the Levodopa-OFF condition (Payoux et al., 2007). In accordance with that, two PET studies (Ceballos-Baumann et al., 1999; Limousin et al., 1997) showed movement-associated activation in the PMC, dorsal PFC and around the SMA (ipsilateral to stimulation and contralateral to movement) during DBS-STN.

A series of NIRS neuroimaging experiments for detecting the characteristics of brainwaves under different conditions have appeared in the past and during recent years. A wearable, multi-channel fNIRS imaging system was presented and tested in $8 \mathrm{HC}$ subjects while riding bicycles outdoors (Piper et al., 2014). In HC subjects, the evoked activity of simple hand movement increased linearly in the SM1 and SMA in images from a NIRS (Derosière et al., 2014; Wilson et al., 2014). A recent pilot study (Morishita et al., 2016) showed increased activity in motor-associated cortical activity following unilateral Globus Pallidus pars interna (GPi) DBS surgery measured by a fNIRS (ON vs. OFF condition in 6 PD patients). Cortical activity was relatively increased in the contralateral primary motor area and the prefrontal cortex. In another study, high frequency $(120 \mathrm{~Hz})$ GPi stimulation consistently increased oxygenated hemoglobin (oxyHb) with a decrease of deoxyHb in an intensity and time dependent manner in a NIRS imaging of the frontal lobe (Sakatani et al., 1999). In addition to the improved motor symptoms of patients with PD, DBS-STN may alter emotional evocation, as it was demonstrated that the STN is correlated with unique patterns of NIRS oxy-Hb changes in PFC during an emotion evocation task (Bick et al., 2016). NIRS shows bilateral activations in the SM1, SMA and PM during walking (Miyai et al., 2001; Perrey, 2014). Levodopa PD ON had a higher oxyHb concentration in the frontal lobe during usual walking, as measured by 2 probes placed on the subject's forehead (Maidan et al., 2016).

To our knowledge, no study has directly examined DBSSTN related changes in cortical activity specifically during gait and FT tasks in images of a multichannel NIRS. We hypothesized the following: for PD patients, the cortex activity in the ON state for both FT and gait will be higher than in the OFF state.

\section{Materials and methods}

\section{Study participants and neurological assessment}

We included 9 patients with advanced PD (1F, 8M, median age: 65 , interquartile range: 6.3 yrs.), chronically treated with double-sided DBS-STN with a quadripolar electrode (Medtronic DBS system, Fridley, Minnesota, USA). Participants were recruited from a randomized control study on DBS. The study was approved by the Ethics Committee of the General University Hospital in Prague, and therefore performed in accordance with the ethical standards established in the 1964 Declaration of Helsinki. A written, informed consent was obtained prior to data collection. The clinical status of the patients was expressed through the unified Parkinson's disease rating scale (UPDRS), part III: clinician-scores monitored the motor evaluation. Akinesia, rigidity and tremor were assessed as subscore items of UPDRS-III. Patients were examined in two sessions after an overnight withdrawal of dopaminergic medication in the DBSSTN OFF state (UPDRS-III: $53.4 \pm(S D)$ 11) and DBS-STN ON at $130 \mathrm{~Hz}$ (UPDRS-III: $29.1 \pm(S D)$ 11.5). The order and the interval of days between examinations were randomized. A $1.5 \mathrm{~h}$ interval was considered sufficient for the onset of full effects and to become accustomed to the frequency change. DBS electrodes were bilaterally implanted using standard stereotactic methods and incorporating intraoperative microelectrode recording. The examinations were performed at least one year after the DBS implantation.

\section{NIRS data acquisition and tasks design}

Red and near-infrared light in the wavelength range from $650 \mathrm{~nm}$ to $900 \mathrm{~nm}$ propagates more deeply into living tissue than visible light of wavelengths shorter than $650 \mathrm{~nm}$, thus hemodynamics in the living body can be non-invasively monitored by using light of this wavelength range where the absorption characteristics of oxyHb and deoxyHb in the blood are different (Scholkmann et al., 2014).

Optical signals are strongly attenuated when passing through biological tissue. The intensity decreases by several orders of magnitude at the distance of several centimeters. A physical model derived from the modified Lambert-Beer law was used for the purpose of determining the changes in oxyhemoglobin concentrations from raw centered signals. The following equation can be defined based on knowledge of recorded time series for 2 wavelengths and extinction coefficient 
values for 2 chromophores ( $\mathrm{HbO}$ and $\mathrm{HbR}$ ) (Cope and Delpy, 1998):

$\Delta c_{H b O}=\frac{e_{H b R, \lambda_{1}} \frac{\Delta A_{\lambda_{2}}}{L_{\lambda_{2}}}-e_{H b R, \lambda_{2}} \frac{\Delta A_{\lambda_{1}}}{L_{\lambda_{1}}}}{e_{H b R, \lambda_{1}} e_{H b O, \lambda_{2}}-e_{H b R, \lambda_{2}} e_{H b O, \lambda_{1}}}$

$c_{\mathrm{HbO}}$ denotes the $\mathrm{HbO}$ concentration, $e_{i}$ represents the extinction coefficient of the given chromophore for a particular wavelength (Table 1$), A_{i}$ corresponds to the raw signal and is proportional to the negative logarithm of the ratio of detected and emitted light intensity, $L_{i}$ represents the mean free path length describing the most likely path of a photon towards the detector (set to $3 \mathrm{~cm}$ ). In a similar way, the equation can be defined for the deoxyhemoglobin.

Cortical activity was acquired simultaneously in 22 channels using a portable NIRS system (NIRSport by NIRX Medizintechnik, Berlin, Germany, sampling frequency $=8.93 \mathrm{~Hz}$ ), which consists of 7 sources and 8 detectors in a head cap. The
Table 1. Values of extinction coefficients $\left(\mathrm{M}^{-1} \mathrm{~cm}^{-1}\right)$ used to calculate the time series of $\mathrm{HbO}$ and $\mathrm{HbR}$ concentrations for the NIRS device (taken from the device documentation)

\begin{tabular}{cl|cc}
\hline \multicolumn{2}{c|}{$760 \mathrm{~nm}$} & \multicolumn{2}{c}{$850 \mathrm{~nm}$} \\
\hline $\mathrm{HbO}$ & $\mathrm{HbR}$ & $\mathrm{HbO}$ & $\mathrm{HbR}$ \\
1.49 & 3.84 & 2.53 & 1.8 \\
\hline
\end{tabular}

NIRS usually incorporates lasers or light-emitting diodes as a lights source. The device emits NIR radiation at 2 wavelengths, 760 and $850 \mathrm{~nm}$. Photodetectors were arranged on the scalp using the international 10-20 EEG system for positioning: F1, FC1, CP1, FC3, F2, FC2, CP2, FC4 (Fig. 1A). An accurate 3D neuroanatomic position is available through the coordinate atlas of the MNI standard (Montreal Neurological Institute). The NIRS cap was placed so that the source $C z$ was located halfway between the nasion-inion. We chose to use NIRS because it is noninvasive, compatible with implanted electrodes and has good motion tolerance for tremors.
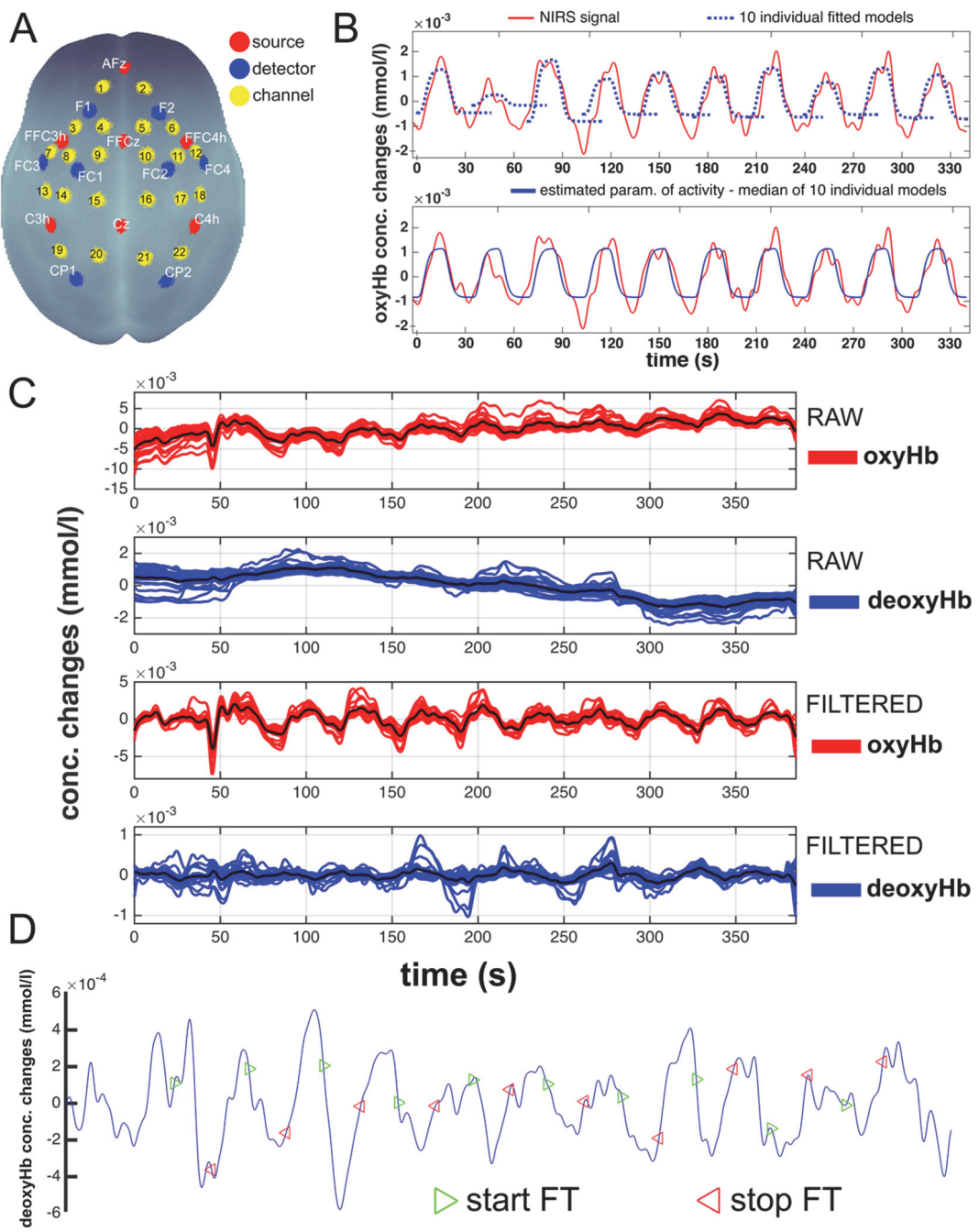

Fig. 1. (A) Scheme of the spatial distribution of detectors, sources and channels above cortical regions of the brain with respect to the EEG $10-20$ system. 22 channels covered frontal cortex to parietal areas of the cortex. (B) NIRS signal for one patient in the ON state (red), for 10 repetitions of finger tapping and resting periods. The top panel additionally shows the hemodynamic functions fitted to the individual repetitions (blue, dashed lines) and the lower panel shows median time course computed from 10 repetitions (blue, solid line). (C) Raw finger tapping data from a single representative subject from all the channels in the DBS ON state showing oxy and deoxy responses before and after filtering. (D) Deoxygenated hemoglobin signal is characterized by much lower amplitudes compared to oxyHb. 
Each task was repeated 10 times continuously in a block diagram (Fig. 2):

a) For the right hand FT task, each cycle consisted of 15 seconds of alternating finger motion and 15 seconds of rest. We tested finger tapping for the right hands (more severely affected right sides were observed for all patients). b) For gait, each cycle comprised of about 12 seconds including the gait phase (starting from standing) and about a 60 seconds break, including sitting down, resting on a chair, rising from the chair and a few seconds for stabilization before the next gait cycle.
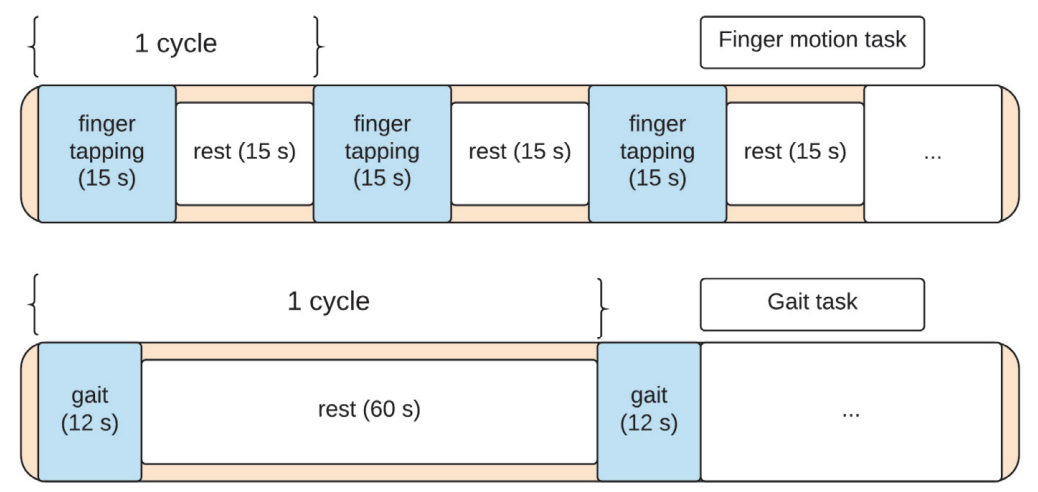

Fig. 2. An illustrative diagram of sessions in the NIRS experiment. The NIRS experiments consisted of two sessions. Each session was a block design consisting of ten resting states alternated with ten motion task states.

\section{Finger tapping movement measurement}

For the purpose of objectively comparing motor changes in finger tapping, we simultaneously measured FT subtest of the UPDRS-III (item 23: the patient taps the index finger on the thumb as quickly and as extended as possible). The movement characteristics were measured by the Optitrack V120 : Trio (NaturalPoint, Inc., Corvallis, USA) validated by (Krupicka et al., 2017). The system measured the spatial position of the markers placed on the first knuckle of the index finger and the first knuckle of the thumb. The mutual Euclidean distance of markers were used for computation of FT PD relevant movement parameters:

a) The finger tapping frequency $[\mathrm{Hz}](\mathrm{FRQ})$.

b) Maximum opening velocity $[\mathrm{cm} / \mathrm{s}](\mathrm{VELO})$ as a mean of maximum opening velocities in all tapping cycles.

c) The power of tapping (POW) as an integral of the tapping signal.

\section{Walkway system assessment}

Gait was assessed using a $5.15 \mathrm{~m}$ long and $0.9 \mathrm{~m}$ wide instrumented walkway (Platinum model GAITRite ${ }^{\circledR}$, CIRSystem Inc., Franklin, USA) placed $2.43 \mathrm{~m}$ from chair in the middle of the straight gait walkway. The walking trial had a length of $10 \mathrm{~m}$ in a straight line, with a GAITRite positioned in the middle. Processed spatio-temporal gait characteristics are: velocity $(\mathrm{cm} / \mathrm{s})$ - mean velocity of walking (distance travelled per time), cadence (steps/min), step length (cm).

\section{Statistical analysis and signal processing}

Raw data were processed offline using Matlab (MathWorks, Natick, Massachusetts, USA). Changes in (de)oxyHb concentrations were calculated from a demeaned NIRS signal using a modified Beer-Lambert transformation (1). The signals were filtered in a range from 0.015 to $0.3 \mathrm{~Hz}$ to attenuate global drifts and extracerebellar artifacts (Fig. 1C) generated by respiration and cardiac variation (3rd order Butterworth band-pass filter) (Fazli et al., 2012; Tak and Ye, 2014). We have adapted the standard version of the General Linear Model to estimate neuronal activity, i.e. the amplitude of the BOLD signal for each channel - each cycle was separately fitted with a physiologically based Boynton Hemodynamic Response Function in the sense of least mean squares to minimize difference between obtained response and desired model-based response (Fig. 1B). Finally, the median amplitude from the 10 individual cycles was calculated and input for statistical comparison between DBS-STN states ON and OFF (2-sided Wilcoxon paired test; uncorrected). An alpha of 0.05 was used as the cutoff for significance. The NIRS signal (typically $\mu \mathrm{mol} / \mathrm{l}$ ) corresponded to the area under curve (AUC) of the $\mathrm{HbO} / \mathrm{HbR}$ concentration variation during the duration of the movement (Fig. 1C, 1D). The total number of automatically processed recordings was nearly 1600 (oxyHb, deoxyHb, 22 channels, 2 conditions, 9 patients, 2 tasks). When applicable, Cohen's d analyses were done in order to estimate the effect size (via effsize package in R programming language). The magnitude was assessed using the thresholds, i.e. $|\mathrm{d}|<0.2$ = negligible, $|\mathrm{d}|<0.5=$ small, $|\mathrm{d}|<$ $0.8=$ medium, and otherwise large.

\section{NIRS brain mapping}

The NIRS Brain Mapping tool was implemented on top of Matlab based on the knowledge of accurate neuroanatomical standardized MNI coordinates for individual channels. The NIRS Brain Mapping tool is based on triangulation-cubic interpolation of estimated activity values $(Z)$ in channels according to MNI $(X, Y)$ coordinates and fits a hypersurface of the form:

$$
v=f(X, Y, Z)
$$

Anatomical mapping of NIRS channels to EEG $10-20$ positions was evaluated by an expert and can be performed according to Table 2 . 
Table 2. Result of expertise mapping of NIRS channels to anatomical brain areas

\begin{tabular}{lc}
\hline Brain area & EEG 10-20 \\
NIRS channel
\end{tabular}

\section{Results}

NIRS examinations have been shown to be appropriate, safe and clinically feasible. Complications in using the system to measure FT and gait were not observed. The wearable multi-channel NIRS system ran without technical problems for all nine subjects throughout all experimental conditions.

\section{Increased cortical activity during FT in DBS-STN ON state}

The measurement area covered the bilateral cortex, since we expected the left hemisphere to be activated by right hand FT. Investigation of spectroscopic parameters in 9 patients during FT revealed differences of the oxyHb NIRS signal in different cortical regions in different conditions (ON vs. OFF). For both conditions, the average activity in all channels increased in comparison to the resting phase $(p<0.01)$. Interpolated maps (Fig. 3A) show a qualitatively similar pattern of activity in the $\mathrm{ON}$ and $\mathrm{OFF}$ state. Nevertheless, the differential map (ONOFF) pinpoints to statistically significant activated areas associated with planning and execution of movements (Fig. 4). The DBS-STN stimulators influenced activity on both sides but were more pronounced on the contralateral side of the used hand. The correlations between spectroscopic and clinical parameters were not significant. We have not observed any significant changes in deoxyHb responses.
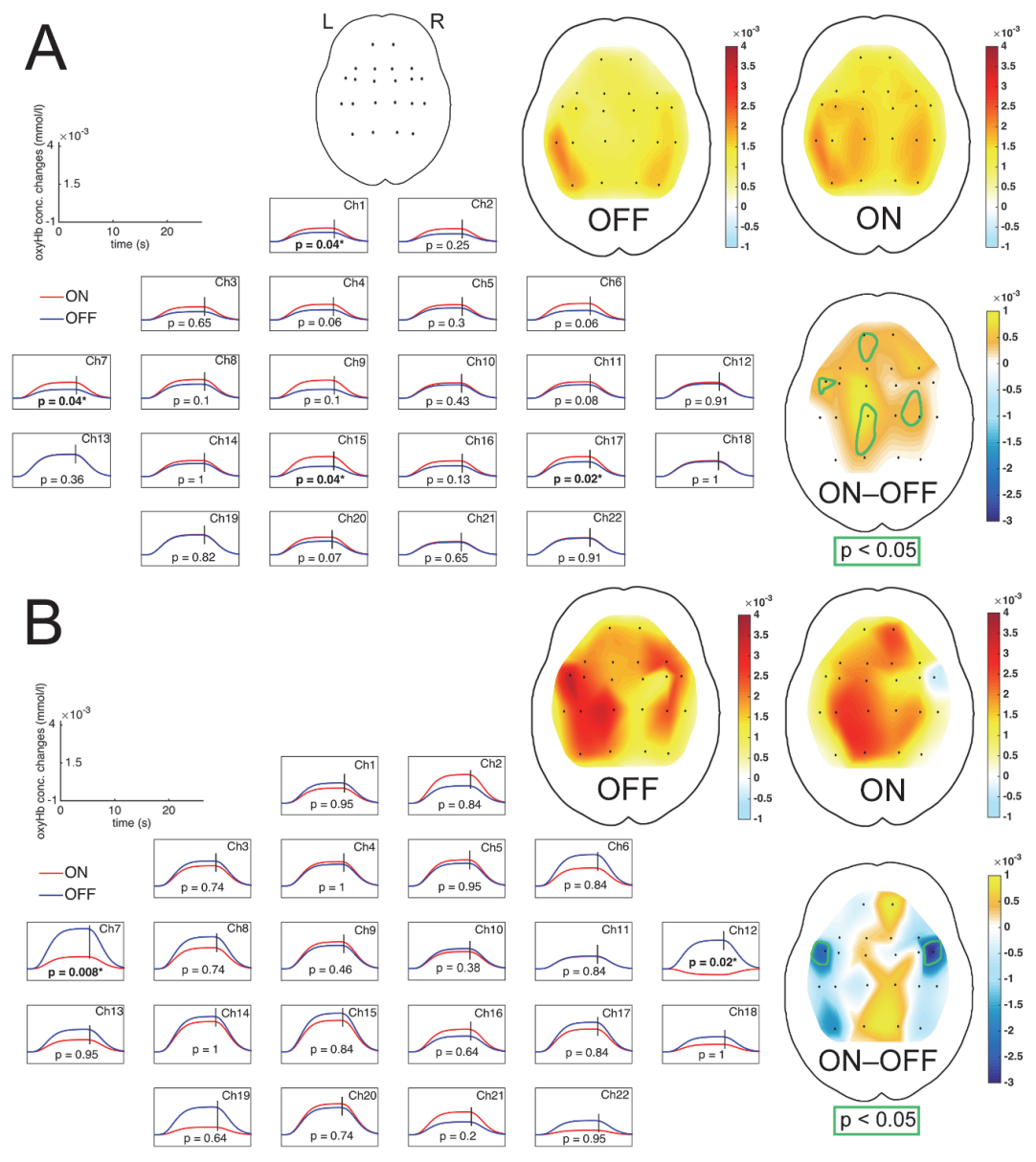

Fig. 3. NIRS brain mapping: Map of average concentration changes (oxygenated hemoglobin) in DBS ON vs. OFF state. The black lines indicate the time of maximum amplitude of hemoglobin concentration. (A) Right hand finger tapping. (B) Gait. The difference between DBS OFF and ON is caused by a decrease in channel 7 and 12 during gait, and the ON map appears to be more focused (centered) above the vertex. 


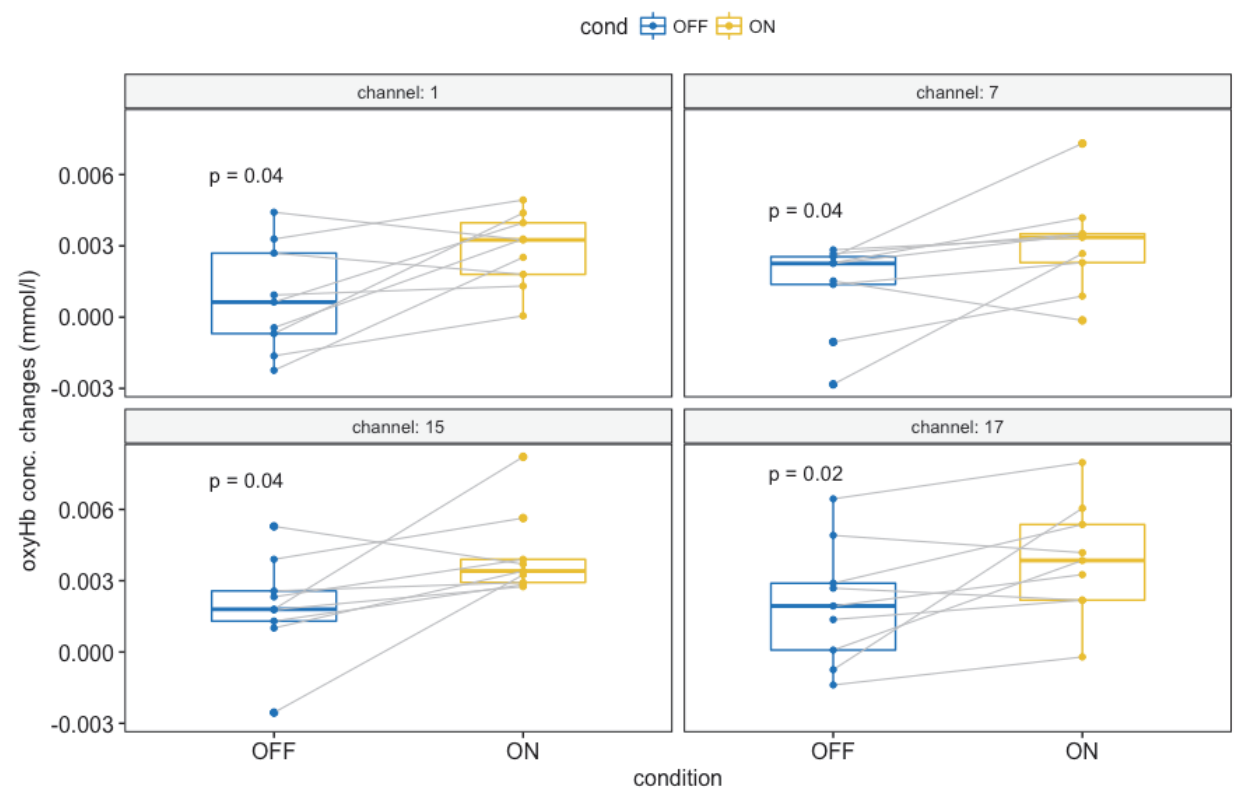

Fig. 4. Paired box plots with significant FT channels.

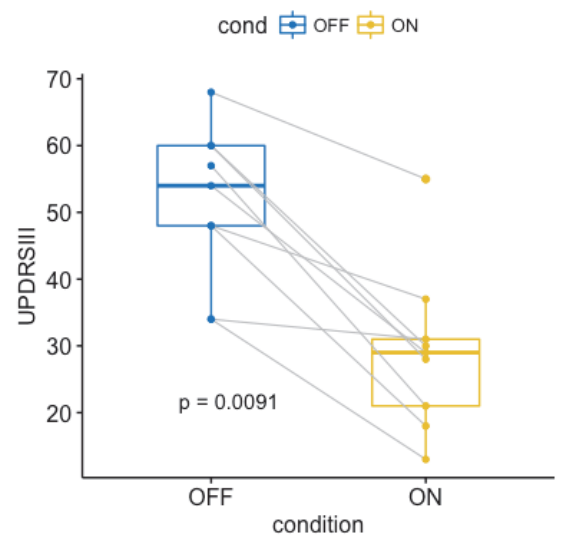

Fig. 5. Paired box plot with change in UPDRS-III. DBS-STN led to the improvement in the overall clinical status.
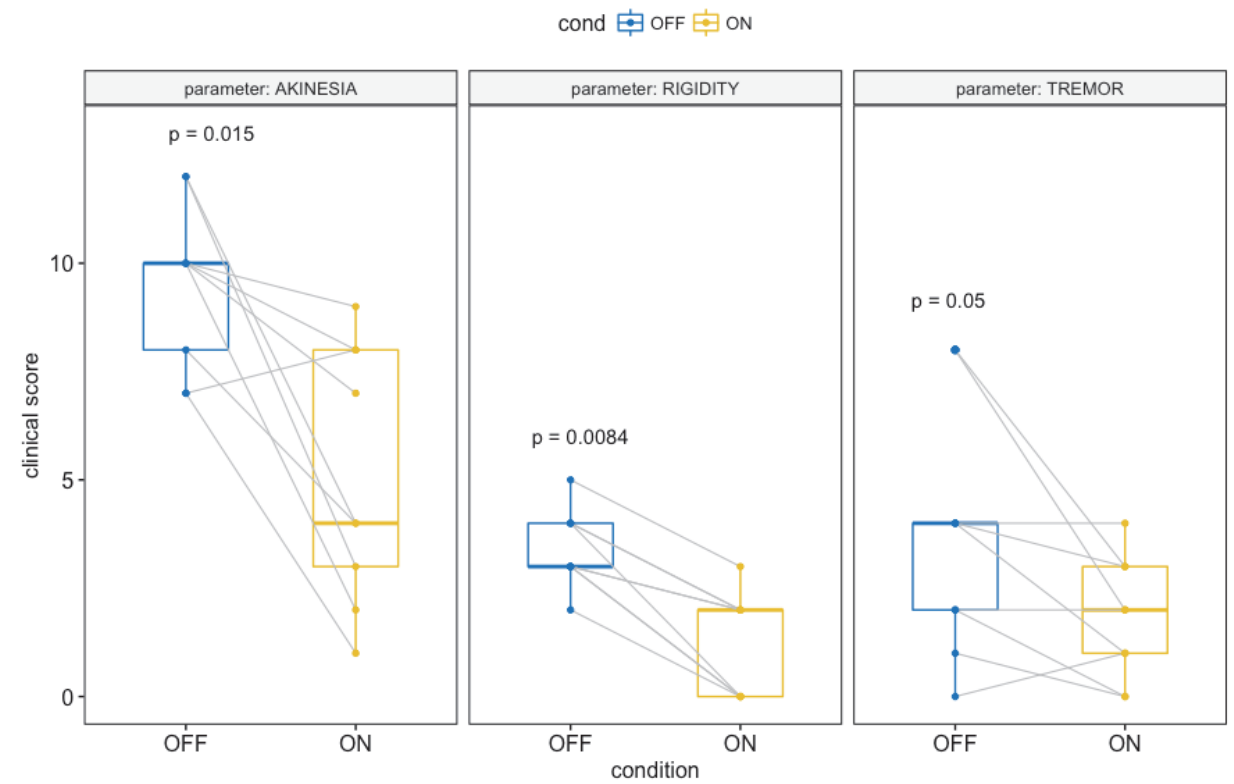

Fig. 6. Paired box plots with changes in UPDRS-III subscores. 
cond 宛 OFF 亩 ON

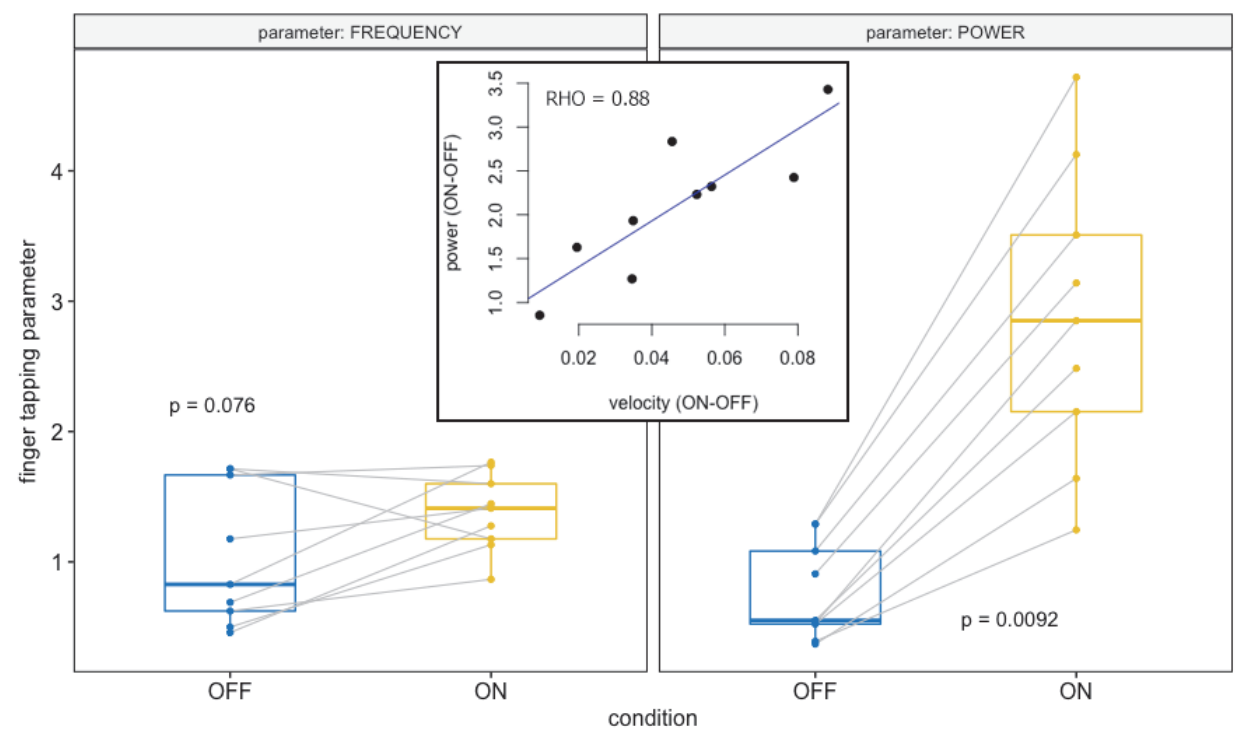

Fig. 7. Paired box plots capturing results from the motion capture camera. DBS-STN led to an improvement in finger movement characteristics. In addition, in the nested graph, finger opening velocity correlates with signal power. The change of frequency was not significant.

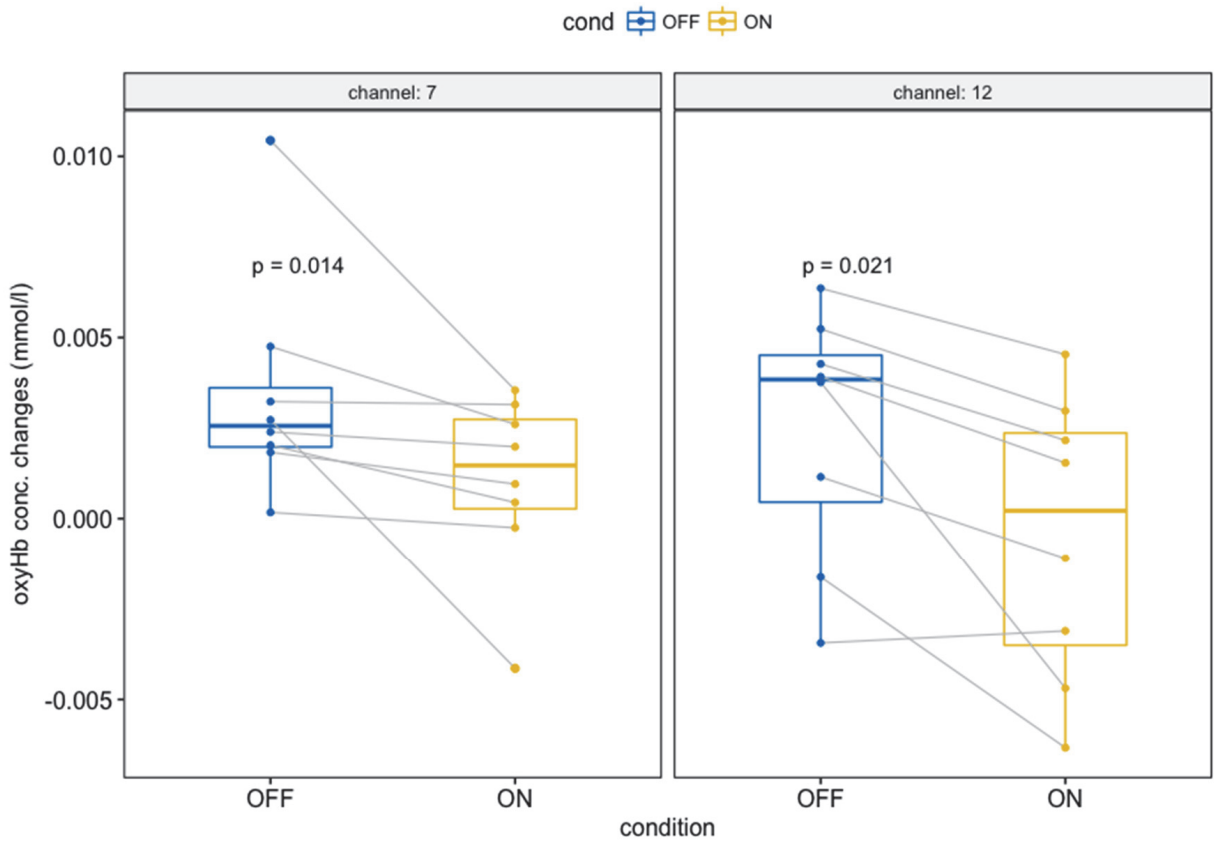

Fig. 8. Paired box plots with significant gait channels.

Regarding UPDRS-III ( $p=0.009$; Fig. 5) scores, statistical analyses indicated significant effects of DBS-STN (Fig. 6): AKINESIA ON versus OFF ( $p=0.015)$, as well as the RIGIDITY $(p=0.008)$. These results show that STN DBS helped to reduce motor symptoms in participants.

Regarding motion capture analysis (higher value = better movement), concordant results were obtained (Fig. 7). The change in stimulation mainly affected the signal power $(p<0.01)$ and opening velocity $(p<0.01)$, despite finger tapping frequency $(p=0.076)$. The correlation between velocity and power was significant and strongly positive (Spearman's rho $=0.88, p=0.003$ ).
Overall, the significant increases in the oxyHb ON state as compared to the OFF state of the stimulator were found in the following channels (Fig. 3A, Fig. 4):

(1) The contralateral intermediate frontal area (AFz-F1, Ch1): Cohen's effect size value $(d=0.84)$ suggests a high practical significance;

(2) The contralateral PMC and Supplementary Motor Cortex (SMC) (FFC3h-FC3, Ch7): Cohen's effect size value $(d=0.77)$ suggests a medium to high practical significance;

(3) The contralateral M1 (Cz-FC1, Ch15): Cohen's effect size value $(d=0.81)$ suggests a high practical significance; 
(4) The ipsilateral PMC and M1 (C4h-FC2, Ch17): Cohen's effect size value $(d=0.76)$ suggests a medium to high practical significance;

(5) It is also to be noted that, on an individual level, the majority of participants followed all the trends mentioned above.

\section{More localized activity during gait in DBS-STN ON state}

Gait recordings from 8 patients were investigated. The other (one remaining patient) contained 3 short-term FOG and was excluded from analysis after video assessment. We did not register any quantitative signs of the effects of the short-term FOG on gait in the NIRS image.

The average DBS-STN ON state oxyHb activity was more localized towards the longitudinal fissure compared to the OFF state (Fig. 3B). For both oxyHb conditions, the average activity against the resting phase was elevated in almost all channels $(p<0.01)$ (with the exception of the Ch12 in the ON condition). The significant highest activity in the OFF state was associated with the PMC and the SMC on both sides (FFC3hFC3, FFC4h-FC4; $p<0.05$ ). No differences in other regions were found. We observed twice the activity values during gait in comparison with FT. Responses for deoxyHb were not significantly affected by DBS switched on.

The GAITRite system in the ON state vs. the OFF produced a longer left-sided step length $(71.9 \mathrm{~cm} \pm 7.0 \mathrm{vs} .66 .2 \mathrm{~cm} \pm$ $9.7, p=0.008)$ and right-sided step length $(72.4 \mathrm{~cm} \pm 6.2 \mathrm{vs}$. $66.1 \mathrm{~cm} \pm 10.3, p=0.008)$. The change in cadence was not significant (122.7 steps/min \pm 10.4 vs. 121.6 steps $/ \mathrm{min} \pm 12.7$, $p=0.55)$, as well as no change in normalized velocity (1.59 \pm 0.28 vs. $1.45 \pm 0.35, p=0.08)$.

Overall, a statistical difference in the oxyHb ON state as compared to the OFF state of the stimulator was found in the following (Fig. 3B, Fig. 8):

(1) The left PMC and the SMC (FFC3h-FC3, Ch7): Cohen's effect size value $(d=0.70)$ suggests a medium to high practical significance;

(2) The right PMC and the SMC (FFC4h-FC4, Ch12): Cohen's effect size value $(d=0.62)$ suggests a medium to high practical significance.

\section{Discussion}

We propose a measurement protocol for capturing motor cortex activity patterns in a wearable, multi-channel fNIRS imaging system in patients with PD. We show how the required measurement protocol can be implemented with simultaneous measurements of finger movements with a motion capture camera and the GAITRite walkway system. These parallel measurements allow for the alignment of neuroimaging and biomechanical methods. We investigated cortical activity changes in both hemispheres of patients with PD that were treated with bilateral DBS-STN. As expected, our results show an increase in NIRS activity against the resting state during FT and gait.

The primary finding in the domain is the new way to measure the change in cortical activity induced by the subthalamic DBS, where left-sided motor and sensorimotor regions were more activated during tapping with the right upper limb. We do not know whether this is a sign of normalization or compensation, because we did not measure healthy controls. However, we feel that our discovery - namely, that these changes took place in the motor region of the contralateral hemisphere - is quite significant. We can exclude that our results are driven by global extra-cerebral noise because only very few channels but not most of them show significant differences. Furthermore, these channels are located in expected regions of the somatomotoric cortex and were filtered by the Butterworth band-pass filter (Fazli et al., 2012; Tak and Ye, 2014). We have to further mention that we present the contrast between two states (DBS-ON and DBS-OFF). Under the assumption of equal global noise between these two states, global hemodynamics were thereby subtracted.

A very perspective method proposed to separate the global and local components in functional near-infrared spectroscopy signals is the principal component spatial filtering (Zhang et al., 2016). Nevertheless, as it is not yet fully integrated into the standard work flow for NIRS analysis and the algorithm might exclude small portions of actual neuronal activity, i.e. it might be too conservative. The effect of this method can be the topic of further research, specifically with focus on the effect of the spatial filtering on gait.

Our second significant finding is the change in cortical activity during gait, when it was demonstrated paradoxically with the opposite phenomenon, such as a decrease in the ON activity in the lateral cortex, which can be seen as a focusing of previously diffusive activity over a region in the vertex that is known to somatotopically correspond to the lower limbs. We have found that DBS-STN has a varied influence on the activity of the motor cortex. Higher motor cortex activity shown in the DBS OFF state compared to ON state may reflect an impairment of gait control in patients with $\mathrm{PD}$. We emphasize the main advantage over PET and a fMRI in that is real, not imagined, gait. The seemingly contradictory change, the decrease in oxygenation, does not necessarily mean a different pathophysiological mechanism as opposed to its increase in finger tapping. This can be a plastical change of the cortex associated with consolidating activity into a smaller area. The higher activity values for the gait condition are not surprising though gait implies much more movement than just finger tapping.

Nevertheless, deoxyhemoglobin did not show any significant effects. It is debated that deoxygenated hemoglobin is more stable in comparison to oxygenated hemoglobin but also has much lower amplitudes. E.g. a study by Fazli et al. (2012) on classification accuracy of finger tapping (right vs left) showed equal results for both chromophores (71.1\% and $73.3 \%)$. We therefore rely on oxygenated hemoglobin dynamics. The study of Morishita et al. (2016) accurately shows the status of pre and post DBS-GPi and the differences were also observed only using oxyHb signals. Very low amplitude deoxyHb signals remain unaffected across the entire measured cortex.

DBS-STN allows higher activity in the motion tasks (number of finger taps or change in cadency etc.). Subsequently, higher performance of the movement was reflected in increased NIRS activity. We did not control for changes in FT or gait kinematics of DBS-ON versus DBS-OFF. Thus, we cannot rule out the influence of these factors on obtained NIRS images. These factors were generally different between neuromodulation conditions and thus, by themselves, can explain differences in oxyHb between conditions. For this reason, we cannot confirm that higher NIRS activity were due to higher movement activity or e.g. higher dopamine levels.

We showed that DBS-STN alters contralateral cortical hemodynamics during a simple FT motor task. Our results of contralateral activation during FT confirmed and outperformed earlier findings (Morishita et al., 2016) in a smaller sample with 6 patients, in terms their results were not con- 
sidered statistically significant and involved GPi stimulation solely. Moreover, our FT results are consistent with previous fMRI/PET/NIRS studies. Our data show a relative increase in activity in the motor areas going along with UPDRS-III clinical improvement as shown by changes in oxyHb levels for FT task. In other words, we observed a significant reduction in clinical UPDRS-III score between ON vs OFF (Fig. 5) while NIRS activity increased with DBS ON (Fig. 4).

Assessments of brain activity during complex tasks, like gait, are unsuited for both $\mathrm{FMRI}$ and PET, and can only be investigated with motor imagery (Peterson et al., 2014). In a previous NIRS study in healthy individuals, gait led to increased activity primarily in the prefrontal and supplementary motor area (Koenraadt et al., 2014). Currently, the measurement of NIRS during gait in patients with PD takes place with a small 2-probe system that captures frontal areas. In comparison with Maidan et al. (2016), where patients were examined during complex walking for 30 seconds in the ON state, we provide information about changes in oxyHb concentrations during gait for both conditions (DBS-STN ON and OFF), as measured in 22 channels covering the frontal, central and parietal cortical areas.

Despite promising results, this study had some limitations that must be taken into consideration when interpreting the findings of this study. First, this investigation was conducted with a small number of participants (nine PD patients). Generalization of these results must, therefore, be viewed with caution and the large inter- and intra-variability in movement disorders. The primary reason of this poor recruitment was that a large proportion of the patients were excluded because they did not meet the inclusion criteria for appropriate documentation and all the measurements. Second, more patients should be recruited to confirm the obtained results in future studies, but it is challenging both in terms of measurement and DBS implantation. In addition, a larger population could also facilitate a more in-depth investigation of correlations with clinical status and motion capture systems. Regarding uncorrected results - the Bonferroni correction using the classical interpolation is too conservative (Ye et al., 2009) and would suppress all results as non-significant in this study - a correction is generally needed for mutually independent channels. In contrast, the channels in our study are spatially dependent and interacting on overlapping areas. As part of the publicly-available NIRS processing toolboxes, the uncorrected curation is used by default, e.g. in FC-NIRS (Xu et al., 2015), NIRS-SPM (Ye et al., 2009), and even in other NIRS studies with multiple subjects (Bae et al., 2017; Chang et al, 2014).

\section{Conclusions}

This is the first pilot study describing brain activity patterns obtained with multichannel neuroimaging using NIRS during gait in patients with PD. We found that DBS-STN performed during simple FT motor tasks led to an increase in the activity of the contralateral motor cortex areas. DBS ON state decreased activity more in lateralized areas but increased activity near the longitudinal fissure during gait. Our study demonstrates the utility of NIRS in detecting functional changes of the motor cortex in patients with PD and indicates its future use for applications that are not suited for PET and fMRI. Our findings of altered motor cortex activity during finger tapping task and gait contributes to our understanding of the pathophysiological mechanisms of movement control in PD and the function of DBS-STN stimulators. In general terms, the pres- ent study demonstrates the potential utility of the NIRS method in detecting functional changes of the brain during gait in patients with PD and other movement disorders.

\section{Conflict of interests}

The authors declare no conflict of interests in this article.

\section{Acknowledgements}

This work was supported by the Czech Science foundation GACR 16-13323S; the Czech Ministry of Health (AZV Grant no. 16-28119a and no. 17-32318a).

\section{References}

Bae SJ, Jang SH, Seo JP, Chang PH (2017). The optimal speed for cortical activation of passive wrist movements performed by a rehabilitation robot: a functional NIRS study. Front Hum Neurosci 11: 194. DOI: 10.3389/fnhum.2017.00194.

Bick SK, Folley BS, Mayer JS, Park S, Charles PD, Camalier CR, et al. (2016). Subthalamic nucleus deep brain stimulation alters prefrontal correlates of emotion induction. Neuromodulation 20(3): 233-237. DOI: 10.1111/ner.12537.

Blinkenberg M, Bonde C, Holm S, Svarer C, Andersen J, Paulson OB, Law I (1996). Rate dependence of regional cerebral activation during performance of a repetitive motor task: a PET study. J Cereb Blood Flow Metab 16(5): 794-803. DOI: 10.1097/00004647-199609000-00004.

Boushel R, Piantadosi CA (2000). Near-infrared spectroscopy for monitoring muscle oxygenation. Acta Physiol Scand 168(4): 615-622. DOI: 10.1046/j.1365-201x.2000.00713.x.

Ceballos-Baumann AO, Boecker H, Bartenstein P, Falkenhayn I, Riescher H, Conrad B, et al. (1999). A positron emission tomographic study of subthalamic nucleus stimulation in Parkinson disease: enhanced movement-related activity of motorassociation cortex and decreased motor cortex resting activity. Arch Neurol 56(8): 997-1003. DOI: 10.1001/archneur.56.8.997.

Chang PH, Lee S-H, Gu K-M, Lee S-H, Jin S-H, Yeo SS, et al. (2014). The cortical activation pattern by a rehabilitation robotic hand: a functional NIRS study. Front Hum Neurosci 8: 49. DOI: 10.3389/fnhum.2014.00049.

Cope M, Delpy DT (1988). System for long-term measurement of cerebral blood and tissue oxygenation on newborn infants by near infra-red transillumination. Med Biol Eng Comput 26(3): 289-294. DOI: 10.1007/BF02447083.

Cui X, Bray S, Bryant DM, Glover GH, Reiss AL (2011). A quantitative comparison of NIRS and fMRI across multiple cognitive tasks. Neuroimage 54(4): 2808-2821. DOI: 10.1016/j. neuroimage.2010.10.069.

Derosière G, Alexandre F, Bourdillon N, Mandrick K, Ward TE, Perrey S (2014). Similar scaling of contralateral and ipsilateral cortical responses during graded unimanual force generation. Neuroimage 85: 471-477. DOI: 10.1016/j. neuroimage.2013.02.006.

Fazli S, Mehnert J, Steinbrink J, Curio G, Villringer A, Müller K-R, Blankertz B (2012). Enhanced performance by a hybrid NIRSEEG brain computer interface. NeuroImage 59(1): 519-529. DOI: 10.1016/j.neuroimage.2011.07.084.

Giacometti P, Diamond SG (2013). Compliant head probe for positioning electroencephalography electrodes and nearinfrared spectroscopy optodes. J Biomed Opt 18(2): 27005. DOI: 10.1117/1.JBO.18.2.027005.

Gilat M, Shine JM, Walton CC, O'Callaghan C, Hall JM, Lewis SJ (2015). Brain activation underlying turning in Parkinson's disease patients with and without freezing of gait: a virtual reality fMRI study. NPJ Parkinsons Dis 1: 15020. DOI: 10.1038/ npjparkd.2015.20.

Huang C, Chu H, Zhang Y, Wang X (2018). Deep brain stimulation to alleviate freezing of gait and cognitive dysfunction in Parkinson's disease: update on current research and future perspectives. Front Neurosci 12: 29. DOI: 10.3389/fnins.2018.00029. 
Kawashima R, Inoue K, Sugiura M, Okada K, Ogawa A, Fukuda H (1999). A positron emission tomography study of self-paced finger movements at different frequencies. Neuroscience 92(1): 107-112. DOI: 10.1016/s0306-4522(98)00744-1.

Kim HY, Seo K, Jeon HJ, Lee U, Lee H (2017). Application of functional near-infrared spectroscopy to the study of brain function in humans and animal models. Mol Cells 40(8): 523-532. DOI: $10.14348 /$ molcells.2017.0153.

Koenraadt KL, Roelofsen EG, Duysens J, Keijsers NL (2014). Cortical control of normal gait and precision stepping: an fNIRS study. Neuroimage 85: 415-422. DOI: 10.1016/j. neuroimage.2013.04.070.

Krupicka R, Viteckova S, Cejka V, Klempir O, Szabo Z, Ruzicka E (2017). BradykAn: A motion capture system for objectification of hand motor tests in Parkinson Disease. 2017 E-Health and Bioengineering Conference (EHB): 446-449. DOI: 10.1109/ EHB.2017.7995457.

Limousin P, Greene J, Pollak P, Rothwell J, Benabid AL, Frackowiak R (1997). Changes in cerebral activity pattern due to subthalamic nucleus or internal pallidum stimulation in Parkinson's disease. Ann Neurol 42(3): 283-291. DOI: 10.1002/ana.410420303.

Maidan I, Nieuwhof F, Bernad-Elazari H, Reelick MF, Bloem BR, Giladi N, et al. (2016). The role of the frontal lobe in complex walking among patients with Parkinson's Disease and healthy older adults: an fNIRS study. Neurorehabil Neural Repair 30(10): 963-971. DOI: 10.1177/1545968316650426.

Martinu K, Nagano-Saito A, Fogel S, Monchi O (2014). Asymmetrical effect of levodopa on the neural activity of motor regions in PD. PLoS One 9(11): e111600. DOI: 10.1371/journal.pone.0111600.

Miyai I, Tanabe HC, Sase I, Eda H, Oda I, Konishi I, et al. (2001). Cortical mapping of gait in humans: a near-infrared spectroscopic topography study. Neuroimage 14(5): 1186-1192. DOI: 10.1006/ nimg.2001.0905.

Morishita T, Higuchi M, Saita K, Tsuboi Y, Abe H, Inoue T (2016). Changes in motor-related cortical activity following deep brain stimulation for Parkinson's Disease detected by functional near infrared spectroscopy: a pilot study. Front Hum Neurosci 10: 629. DOI: 10.3389/fnhum.2016.00629.

Payoux P, Brefel-Courbon C, Julian A, Durif F, Azulay JP, Blin O, et al. (2007). Motor activity in parkinsonism and levodopa effect: A PET study. J Nucl Med 48(Suppl. 2): 8P.

Perrey S (2014). Possibilities for examining the neural control of gait in humans with fNIRS. Front Physiol 5: 204. DOI: 10.3389/ fphys.2014.00204.

Peterson DS, Pickett KA, Duncan RP, Perlmutter JS, Earhart GM (2014). Brain activity during complex imagined gait tasks in Parkinson disease. Clin Neurophysiol 125(5): 995-1005. DOI: 10.1016/j.clinph.2013.10.008.

Piper SK, Krueger A, Koch SP, Mehnert J, Habermehl C, Steinbrink J, et al. (2014). A wearable multi-channel fNIRS system for brain imaging in freely moving subjects. Neuroimage 85: 64-71. DOI: 10.1016/j.neuroimage.2013.06.062.

Rao SM, Bandettini PA, Binder JR, Bobholz JA, Hammeke TA, Stein EA, Hyde JS (1996). Relationship between finger movement rate and functional magnetic resonance signal change in human primary motor cortex. J Cereb Blood Flow Metab 16(6): 12501254. DOI: 10.1097/00004647-199611000-00020.
Sabatini U, Boulanouar K, Fabre N, Martin F, Carel C, Colonnese C, et al. (2000). Cortical motor reorganization in akinetic patients with Parkinson's disease: a functional MRI study. Brain 123(Pt 2): 394-403. DOI: 10.1093/brain/123.2.394.

Sadato N, Ibañez V, Campbell G, Deiber MP, Le Bihan D, Hallett M (1996). Frequency dependent changes of regional cerebral blood flow during finger movements. J Cereb Blood Flow Metab 16(1): 23-33. DOI: 10.1097/00004647-199601000-00003.

Sakatani K, Katayama Y, Yamamoto T, Suzuki S (1999). Changes in cerebral blood oxygenation of the frontal lobe induced by direct electrical stimulation of thalamus and globus pallidus: a near infrared spectroscopy study. J Neurol Neurosurg Psychiatry 67(6): 769-773. DOI: 10.1136/jnnp.67.6.769.

Scholkmann F, Kleiser S, Metz AJ, Zimmermann R, Mata Pavia J, Wolf U, Wolf M (2014). A review on continuous wave functional near-infrared spectroscopy and imaging instrumentation and methodology. Neuroimage 85: 6-27. DOI: 10.1016/j. neuroimage.2013.05.004.

Strangman G, Culver JP, Thompson JH, Boas DA (2002). A quantitative comparison of simultaneous BOLD fMRI and NIRS recordings during functional brain activation. Neuroimage 17(2): 719-731. DOI: 10.1006/nimg.2002.1227.

Tak S, Ye JC (2014). Statistical analysis of fNIRS data: A comprehensive review. Neuroimage 85: 72-91. DOI: 10.1016/j. neuroimage.2013.06.016.

Tohka J, Foerde K, Aron AR, Tom SM, Toga AW, Poldrack RA (2008). Automatic independent component labeling for artifact removal in fMRI. Neuroimage 39(3): 1227-1245. DOI: 10.1016/j. neuroimage.2007.10.013.

Torricelli A, Contini D, Pifferi A, Caffini M, Re R, Zucchelli L, Spinelli L (2014). Time domain functional NIRS imaging for human brain mapping. Neuroimage 85: 28-50. DOI: 10.1016/j. neuroimage.2013.05.106.

Varriale P, Collomb-Clerc A, Van Hamme A, Perrochon A, Kemoun G, Sorrentino G, et al. (2018). Decreasing subthalamic deep brain stimulation frequency reverses cognitive interference during gait initiation in Parkinson's disease. Clin Neurophysiol 129(11): 2482-2491. DOI: 10.1016/j.clinph.2018.07.013.

Wilson TW, Kurz MJ, Arpin DJ (2014). Functional specialization within the supplementary motor area: a fNIRS study of bimanual coordination. Neuroimage 85: 445-450. DOI: 10.1016/j. neuroimage.2013.04.112.

Wu T, Long X, Wang L, Hallett M, Zang Y, Li K, Chan P (2011). Functional connectivity of cortical motor areas in the resting state in Parkinson's disease. Hum Brain Mapp 32(9): 1443-1457. DOI: $10.1002 / \mathrm{hbm} .21118$

Xu J, Liu X, Zhang J, Li Z, Wang X, Fang F, Niu H (2015). FC-NIRS: A Functional Connectivity Analysis Tool for NearInfrared Spectroscopy Data. Biomed Res Int 2015: 248724. DOI: $10.1155 / 2015 / 248724$.

Ye J, Tak S, Jang K, Jung J, Jang J (2009). NIRS-SPM: Statistical parametric mapping for near-infrared spectroscopy. Neuroimage 44(2): 428-447. DOI: 10.1016/j.neuroimage.2008.08.036.

Zhang X, Noah JA, Hirsch J (2016). Separation of the global and local components in functional near-infrared spectroscopy signals using principal component spatial filtering. Neurophotonics 3(1): 015004. DOI: 10.1117/1.NPh.3.1.015004. 